\title{
HPV testing in patients with low grade cervical cytological abnormalities: a follow up study
}

\author{
C S Herrington, M F Evans, F M Charnock, W Gray, J O’D McGee
}

\begin{abstract}
Aim-To assess the diagnostic performance of human papillomavirus (HPV) analysis in predicting cervical intraepithelial neoplasia (CIN) grades 2 and 3 in patients with persistent low grade cervical cytological abnormalities.

Methods-Cervical smears from 167 women referred for colposcopy with persistent borderline, wart virus or mildly dyskaryotic changes on cervical screening were analysed by Papanicolaou staining, non-isotopic in situ hybridisation and generic and type specific polymerase chain reaction (PCR) amplification of HPV sequences. Follow up was by cytological and, where appropriate, histological analysis.

Results-CIN grade 2 or 3 was identified in 46 patients after a median follow up of 27 months. HPV positivity by both techniques was associated with high grade CIN and with age less than 30 years (median age 33 years). Non-isotopic in situ hybridisation was more predictive but less sensitive than either generic or type specific PCR, but prediction was greater using either molecular technique in women over 30 years of age.

Conclusions-Although the degree of prediction found is of only limited clinical value, the strong association of HPV positivity with both high grade CIN and patient age suggests that further studies of HPV testing in this patient group are warranted.

(F Clin Pathol 1996;49:493-496)
\end{abstract}

Keywords: human papillomavirus, cytology, in situ hybridisation, polymerase chain reaction.

Nuffield Department of Pathology and

Bacteriology

(University of Oxford),

Departments of

Gynaecology and

Cellular Pathology,

John Radcliffe

Hospital,

Oxford OX3 9DU

Correspondence to: Dr C S Herrington,

Department of Pathology,

University of Liverpool,

Royal Liverpool University

Hospital,

Duncan Building,

Liverpool L69 3BX.

Accepted for publication

20 February 1996
In the UK the recommended management of patients with a cervical smear showing moderate or severe dyskaryosis is immediate referral for colposcopic assessment, diagnosis and treatment. The reporting of borderline changes, wart virus changes or mild dyskaryosis is usually accompanied by a request for a repeat smear with referral for colposcopic assessment only if the abnormality persists. ${ }^{1}$ The main reasons for this approach are to avoid "underdiagnosing" high grade lesions by cytological screening and to detect any progression to a higher grade abnormality. It would therefore be useful to distinguish by further evaluation of cytological material those patients with low grade cytological abnormali- ties who have or will develop high grade cervical intraepithelial neoplasia (CIN).

Human papillomaviruses (HPV) are associated with squamous lesions and classification by molecular homology indicates that there are two major groups, anogenital and cutaneous. More specifically, the association of certain HPV types with intraepithelial and invasive neoplasia of the anogenital region, particularly the cervix uteri, has led to the definition of "high risk" (HPV 16 and 18 particularly), "intermediate risk" (HPV 31 and 33 particularly) and "low risk" (HPV 6 and 11 particularly) anogenital types. ${ }^{3}$ This association suggests that the detection of "high" and "intermediate" risk HPV types in patients with low grade cervical cytological abnormalities might predict those patients who have or will develop CIN grade 2 or 3. Moreover, as HPV infection is less common in older women, HPV analysis may be of more value in women over 30 years of age. ${ }^{4}$ Conventional polymerase chain reaction (PCR) techniques for HPV detection are reportedly of limited use for the detection of current high grade lesions, ${ }^{5}$ most likely because of their high sensitivity, "high" and "intermediate" risk HPV types being detectable in a significant proportion of patients with normal cervical smears. ${ }^{67}$ However, there is some evidence that a greater viral load is more associated with CIN grade 2 or 3 and less sensitive techniques such as in situ hybridisation and semi-quantitative PCR have been shown to be more predictive of the presence of such high grade lesions. ${ }^{5}{ }^{7-11}$ Recent studies have also demonstrated the potential utility of hybrid capture techniques in the same setting. ${ }^{12}$

Although "high" and "intermediate" risk HPV types are frequently present in patients with low grade histological lesions, it is possible that women with such lesions may be at greater risk of progression to a high grade lesion. If this were the case, the demonstration of such infection would be of clinical value.

In the present study patients with persistent low grade cervical cytological abnormalities were assessed by molecular HPV analysis of cytological material obtained at initial colposcopy and were subsequently followed cytologically and with biopsy when appropriate. HPV status was correlated with age and with histological diagnosis to assess its diagnostic utility for the detection of CIN grade 2 or 3 .

\section{Methods}

Patients ( $n=167)$ referred to the colposcopy unit, John Radcliffe Hospital, Oxford, with cervical smears showing persistent borderline 
changes, wart virus changes or mild dyskaryosis were recruited prospectively. Demographic data were recorded and routine colposcopic evaluation undertaken. These patients had a median of three abnormal smears (range one to six) over a median of 24 months (range zero to 66). Two cervical smears were taken at colposcopy, each with a separate Aylesbury spatula and one was submitted for routine cytopathological assessment. The other (sterile) was smeared in the conventional way for in situ hybridisation, the spatula tip then being washed in sterile $0.15 \mathrm{M} \mathrm{NaCl}$ to collect cells for PCR analysis. Cervical smears were fixed in $70 \%$ ethanol, air dried and stored at room temperature. The cells in saline were pelleted by centrifugation, digested with proteinase $K$ $(2 \mu \mathrm{g} / \mathrm{ml})$ and stored at $-20^{\circ} \mathrm{C}$ according to the protocol of Bauer et al. ${ }^{13}$ Any biopsy specimens taken were routinely processed and haematoxylin and eosin stained sections examined. Standard cytopathological and histopathological criteria were used for diagnosis. ${ }^{14}$

\section{NON-ISOTOPIC IN SITU HYBRIDISATION}

This was performed by hybridising smears with a cocktail of digoxigenin labelled nicktranslated probes for HPV 16, 18, 31, and 33 using a method of sensitivity of approximately
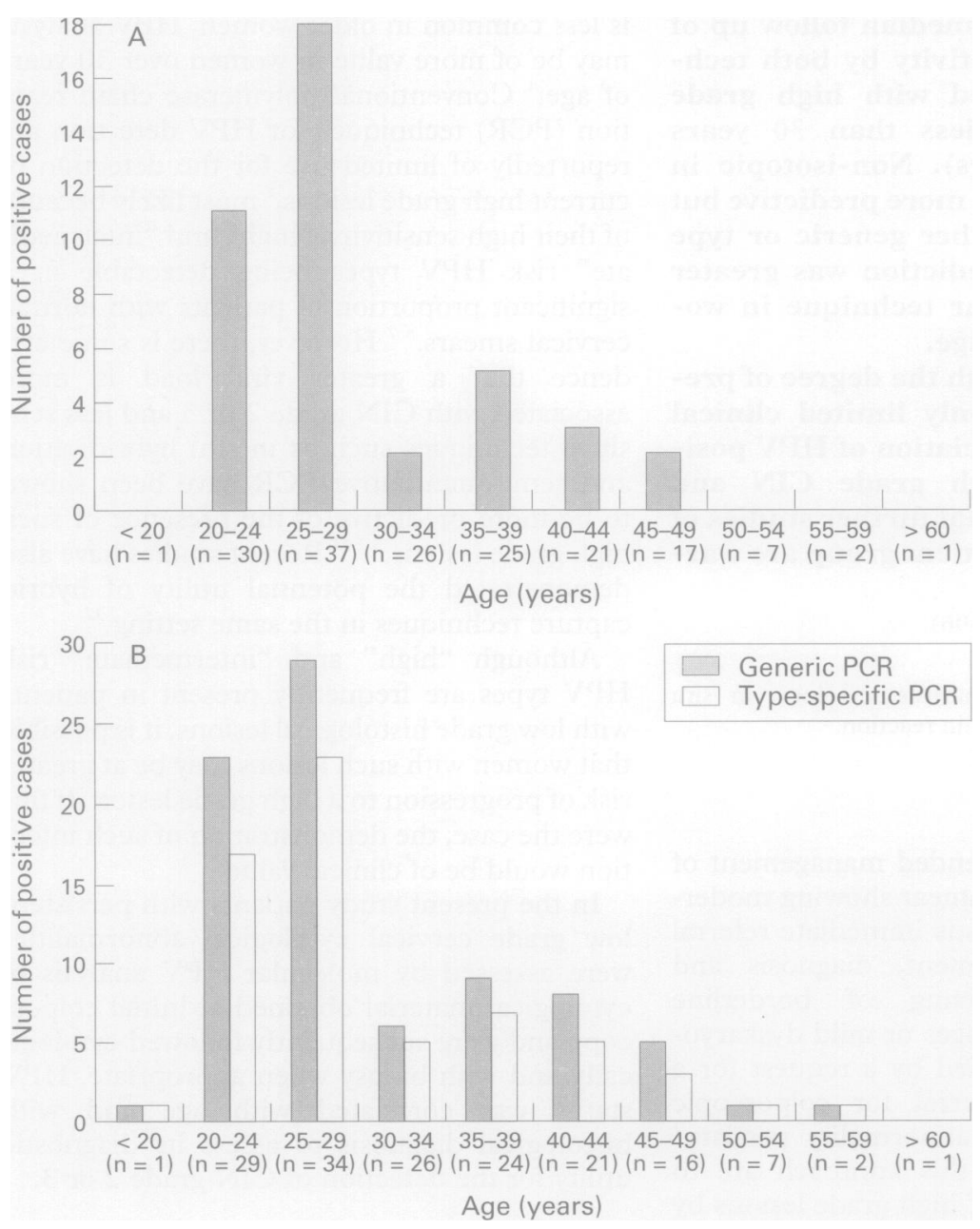

Figure 1 Age distribution of cases containing HPV by $(A)$ non-isotopic in situ hybridisation and $(B)$ generic and type specific PCR. Note that the distributions have the same form. The patients for whom $\beta$-globin did not amplify have been excluded from the $P C R$ data and the type specific PCR data include only "high" and "intermediate" risk HPV types.
2.5-12 copies of integrated HPV per cell as described previously. ${ }^{15}$ CaSki cell smears were used as positive controls.

\section{PCR AMPLIFICATION}

PCR amplification of a 450 base pair (bp) segment of the L1 gene was carried out using degenerate consensus primers by a modification of the method of Bauer $\mathrm{et} \mathrm{al}^{13}$ as described previously. ${ }^{10}$ Products were identified as HPV sequences by dot blot hybridisation with a mixture of degenerate consensus probes which detect a wide range of HPV types. HPV typing was carried out by dot blot hybridisation for HPV $6 / 11,16,18,31$, and $33 .{ }^{10}{ }^{13}$ Plasmid derived PCR products were incorporated as positive controls and reactions carried out in the absence of DNA as negative controls. A 536 bp $\beta$-globin fragment was amplified as an internal control.

PATIENT FOLLOW UP AND DATA ANALYSIS

Patients for whom no follow up was available were excluded from analysis. Patients for whom no $\beta$-globin amplification was obtained were excluded from the analysis of PCR results. Sensitivity, predictive values and likelihood ratios were calculated according to Sackett et $a l .{ }^{17}$ Independent variables were compared using the $\chi^{2}$ test, with Yates' correction where appropriate.

\section{Results}

Biopsy specimens were taken at initial colposcopy from 142 patients and were negative in 14 , showed wart virus changes in $63, \mathrm{CIN}$ grade 1 in $25, \mathrm{CIN}$ grade 2 in 12 , and CIN grade 3 in 28 . No invasive carcinomas were found. After a median follow up of 27 months (range 12-37 months) CIN grade 2 was found on follow up biopsy in two and CIN grade 3 in four further patients, giving a total of 46 patients with CIN grade 2 or 3 . There was a significant assocation between age under 30 years and the identification of CIN grade 2 or 3 (median age 33 years; $\chi^{2}=8.5, \mathrm{DF}=1, \mathrm{p}<$ 0.01 ). Of the remaining 121 patients, seven were lost to follow up, 102 had at least one subsequent normal smear and 13 had persistent low grade abnormalities.

Non-isotopic in situ hybridisation alone was positive in $41(24.6 \%)$ patients and positivity was significantly associated with CIN grade 2 or 3 on biopsy $\left(\chi^{2}=45.2, \mathrm{DF}=1, \mathrm{p}<0.001\right)$. The relation between patient age at the time of colposcopy and the presence of "high" and "intermediate" risk HPV types as determined by non-isotopic in situ hybridisation is shown in fig 1A: patients aged less than 30 were significantly more likely to be HPV positive than those over $30\left(\chi^{2}=20.2, \mathrm{DF}=1, \mathrm{p}<\right.$ 0.001 ), the prevalences being $42.6 \%$ and 12.2 $\%$, respectively. However, the association with CIN grade 2 or 3 was greater in patients over $30\left(\chi^{2}=23.5, \mathrm{DF}=1, \mathrm{p}<0.001\right)$ than in those under 30 years of age $\left(\chi^{2}=13.4, D F=1, p<\right.$ 0.001 ), although both associations were highly significant. The sensitivity, predictive values and likelihood ratios of non-isotopic in situ hybridisation for the presence of CIN grade 2 or 3 are given in table 1. 


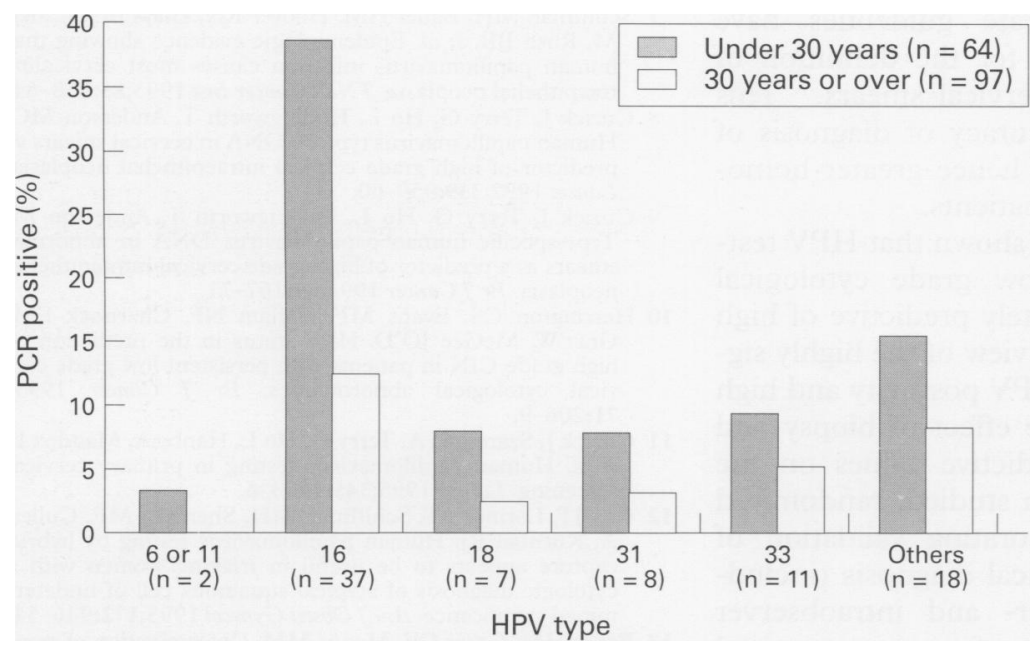

Figure 2 The proportion of patients under 30 years of age $(n=64)$ and 30 years of age or older $(n=97)$ whose cytological samples contained different HPV types on PCR. Those patients for whom $\beta$-globin did not amplify have been excluded.

Table 1 Positive predictive values (PPV) and sensitivities for the detection of CIN 2 or 3 by non-isotopic in situ hybridisation (NISH), generic PCR and type specific PCR for "high" and "intermediate" risk HPV types. Likelihood ratios represent the ratio of sensitivity to (1-specificity). ${ }^{17}$

\begin{tabular}{llll}
\hline & $\begin{array}{l}P P V \\
(\%)\end{array}$ & $\begin{array}{l}\text { Sensitivity } \\
(\%)\end{array}$ & $\begin{array}{l}\text { Likelihood } \\
\text { ratio }\end{array}$ \\
\hline NISH & & & \\
overall & 70 & 61 & 5.8 \\
<30 years & 68 & 70 & 2.9 \\
$\quad$ >30 years & 75 & 47 & 12.2 \\
$\begin{array}{l}\text { Generic PCR } \\
\text { overall }\end{array}$ & 48 & 88 & 2.4 \\
<30 years & 46 & 96 & 1.3 \\
>30 years & 52 & 79 & 4.2 \\
Type specific PCR & & & \\
overall & 54 & 77 & 3 \\
<30 years & 49 & 79 & 1.4 \\
$>30$ years & 64 & 74 & 6.9 \\
\hline
\end{tabular}

$\beta$-globin amplification was not detected in six patients and hence the DNA was not of sufficient quality for HPV analysis by PCR; all of these patients were HPV negative. Of the remaining patients, $83(51.6 \%)$ were positive after hybridisation with the generic HPV probe. Sixty five patients had types $6,11,16$, 18,31 , or 33 , and the remaining 18 cases positive with the generic probe were classified as "others" (fig 2). The two patients with HPV $6 / 11$ were included in the generic PCR results but excluded from the type specific PCR results, which are therefore restricted to "high" and "intermediate" risk types. A high grade lesion on biopsy was significantly associated with positivity by both generic PCR $\left(\chi^{2}=27.5\right.$, $\mathrm{DF}=1, \mathrm{p}<0.001)$ and type specific $\operatorname{PCR}\left(\chi^{2}\right.$ $=31.3, \mathrm{DF}=1, \mathrm{p}<0.001)$. The relation between patient age at the time of colposcopy and HPV as determined by generic and type specific PCR is shown in fig $1 \mathrm{~B}$. There was a significant association between age less than 30 years and HPV positivity by both generic $\left(\chi^{2}=\right.$ 36.0, DF $=1, \mathrm{p}<0.001)$ and type specific $\left(\chi^{2}\right.$ $=27.9, \mathrm{DF}=1, \mathrm{p}<0.001)$ PCR amplification. The sensitivities, predictive values, and likelihood ratios of generic and type specific PCR for the presence of CIN grade 2 or 3 are given in table 1 .

\section{Discussion}

These data confirm the assocation of "high" and "intermediate" risk HPV types, determined by both non-isotopic in situ hybridisation and PCR, with high grade CIN. The use of PCR, which is of greater absolute sensitivity, increases diagnostic sensitivity but reduces the positive predictive value. This is also reflected in the likelihood ratios which are less affected by the prevalence of the underlying disease in the population studied ${ }^{17}$ and therefore give a better estimate of the potential clinical value of HPV testing.

There is a strong correlation between patient age and the presence of HPV DNA determined by both non-isotopic in situ hybridisation and PCR, women less than 30 years of age being significantly more likely to harbour HPV sequences, particularly HPV 16 . Although the less likely possibility of a cohort effect cannot be entirely excluded, the high prevalence of HPV sequences in women under 30 years of age and the relatively low number of patients with HPV6/11 infection is most likely related to the natural history of HPV infection in these patients. Moreover, the magnitude of the prevalence of HPV sequences as determined by generic PCR (82.6\%) suggests that persistent low grade cytological abnormalities are a result of HPV infection in the majority of younger patients.

The higher prevalence of HPV sequences in younger women is parallelled to some degree by an increased prevalence of CIN grade 2 or 3 , but the strength of the association between HPV and age is greater than that between histological grade and age. This suggests that infection with these viral types is reversible, even when productive as assessed by nonisotopic in situ hybridisation. ${ }^{18}$ This hypothesis is supported by the lower positive predictive values and likelihood ratios of HPV testing in women under 30 years of age and is consistent with the finding that persistence of HPV is associated with persistence of intraepithelial neoplasia. ${ }^{19} \mathrm{HPV}$ testing is therefore of greater diagnostic value in women over 30 years of age in this group of patients, although greater predictive value is achieved at the expense of lower sensitivity.

HPV DNA detection in this group of patients therefore seems to be of limited predictive value because of the occurrence of HPV infection in patients who do not have or develop CIN grade 2 or 3 . However, the effect of the biopsy procedure on the subsequent natural history of HPV infection is not known and HPV testing would be of more value in patient management if applied at the time of initial detection of a low grade abnormality. This study may therefore underestimate the predictive potential of HPV testing in patients who have not had a biopsy specimen taken. Similarly, long term follow up of patients with borderline cervical cytological abnormalities has shown that the risk of developing CIN grade 2 or 3 persists up to nine years after the initial cytological abnormality and is greatest in women between 20 and 39 years of age. ${ }^{20}$ 
Moreover, more accurate guidelines have recently been proposed for the definition of borderline changes in cervical smears. ${ }^{21}$ This may lead to greater accuracy of diagnosis of these abnormalities and hence greater homogeneity in this group of patients.

The present study has shown that HPV testing in patients with low grade cytological abnormalities is moderately predictive of high grade CIN. However, in view of the highly significant association of HPV positivity and high grade CIN, the possible effect of biopsy and the dependence of predictive values on the nature of the population studied, randomised controlled trials incorporating validation of cytological and histological diagnosis (including assessment of inter- and intraobserver variation) and estimation of cost are required to assess the role of HPV testing in the management of these patients. The data presented here support such an approach.

We thank the staff of the Colposcopy unit for their help in obtaining samples and Oxford Regional Health Authority and the Cancer Research Campaign (UK) for financial support.

1 Oxford Regional Health Authority. National Co-ordinating Network Report. NHS Cervical Screening Programme. Oxford: Oxford Regional Health Authority, 1992.

2 Herrington CS. Human papillomaviruses in cervical neoplasia I: classification, virology, pathology and epidemiology. $\mathcal{F}$ Clin Pathol 1994;47:1066-72.

3 Lorincz AT, Reid R, Jenson AB, Greenberg MD, Lancaster W, Kurman RJ. Human papillomavirus infection of the cervix: relative risk associations of 15 common anogenital types. Obstet Gynecol 1993;79:328-37.

4 Meijer CILM, van den Brule AJ, Snijders PJ, Helmerhorst T, Kenemans P, Walboomers JMM. Detection of human papillomavirus in cervcal scrapes by the polymerase chain papillomavirus in cervcal scrapes by the polymerase chain reaction in relation to cytology: possible implications for 81 .

5 Bavin PJ, Giles JA, Deery A, Crow J, Griffiths PD, Emery VC, et al. Use of semi-quantitative PCR for human papillomavirus DNA type 16 to identify women with high grade cervical disease in a population presenting with a mildly dyskaryotic smear report. $B r \mathcal{F}$ Cancer 1993;67:602-5.

6 Schiffman M. Recent progress in defining the epidemiology of human papillomavirus infection and cervical neoplasia. 千 Natl Cancer Inst 1992;84:394-8.
7 Schiffman MH, Bauer HM, Hoover RN, Glass AG, Cadel $\mathrm{M}$, Rush BB, et al. Epidemiologic evidence showing that human papillomavirus infection causes most cervicalintraepithelial neoplasia. $\mathcal{F}$ Natl Cancer Inst 1993;85:958-64.

8 Cuzick J, Terry G, Ho L, Hollingworth T, Anderson MC. Human papillomavirus type 16 DNA in cervical smears as predictor of high grade cervical intraepithelial neoplasia. Lancet 1992;339:959-60.

9 Cuzick J, Terry G, Ho L, Hollingworth T, Anderson M. Type-specific human papillomavirus DNA in abnormal Type-specific human papillomavirus DNA in abnormal smears as a predictor of high-grade cervical

10 Herrington CS, Evans MF, Hallam NF, Charnock FM, Gray W, McGee JO'D. HPV status in the prediction of high grade CIN in patients with persistent low grade cervical cytological abnormalities. $\mathrm{Br} f$ Cancer 1995; 71:206-9.

11 Cuzick J, Szarewski A, Terry G, Ho L, Hanby A, Maddox P, et al. Human papillomavirus testing in primary cervical screening. Lancet 1995;345:1533-6.

12 Cox JT, Lorincz AT, Schiffman MH, Sherman ME, Cullen A, Kurman RJ. Human papillomavirus testing by hybrid capture appears to be useful in triaging women with a cytologic diagnosis of atypical squamous cell of undetermined significance. Am $\mathcal{F}$ Obstet Gynecol 1995;172:946-54.

13 Bauer HM, Greer CE, Manos MM. Determination of genital human papillomavirus infection by consensus PCR amplification. In: Herrington CS, McGee JO'D, eds. Diagnostic molecular pathology: a practical approach. Vol 2. Oxford: Oxford University Press, 1992:131-52.

14 Coleman DV, Evans DMD. Biopsy pathology and cytology of the cervix. London: Chapman and Hall, 1988.

15 Herrington CS, de Angelis M, Evans MF, Troncone G, $M c G e e$ JO'D. Detection of high risk human papillomavirus in routine cervical smears: strategy for screening. $\mathcal{F}$ Clin Pathol 1992;45:385-90.

16 Herrington CS, Graham AK, McGee JO'D. Interphase cytogenetics using biotin and digoxigenin labelled probes: III. Increased sensitivity and flexibility for detecting HPV in cervical biopsy specimens and cell lines. 7 Clin Pathol in cervical biopsy

17 Sackett DL, Haynes RB, Guyatt GH, Tugwell P. Clinical epidemiology: a basic science for clinical medicine. 2nd edn. Boston: Little Brown, 1991.

18 Graham AK, Herrington CS, McGee JO'D. Sensitivity and specificity of monoclonal antibodies to human papillomavirus type 16 capsid protein: comparison with simultaneous viral detection by non-isotopic in situ hybridisation. 7 Clin Pathol 1991;44:96-101.

19 Ho GYF, Burk RD, Klein S, Kadish AS, Chang CJ, Palan P, et al. Persistent genital human papillomavirus infection as a risk factor for persistent cervical dysplasia. $\mathcal{f}$ Natl Cancer a risk factor for persistent

20 Hirschowitz L, Raffle AE, Mackenzie EF, Hughes AO. Long term follow up of women with borderline cervical smear test results: effects of age and viral infection on progression to high grade dyskaryosis. $B M F$ 1992;304:1209-12.

21 Buckley CH, Herbert A, Johnson J, Mackenzie EFD, Smith JHF, Smith PA, et al. Borderline nuclear changes in cervical smears: guidelines on their recognition and management. F Clin Pathol 1994;47:481-92. 